FREIBURG-PHENO-2010-021, IPPP/10/51, DCPT/10/102

\title{
Recent Progress in the Golem Project
}

Gavin Cullen $^{\mathrm{a}}$, Nicolas Greiner ${ }^{\mathrm{b}}$, Alberto Guffanti ${ }^{\mathrm{c}}$, Jean-Philippe Guillet ${ }^{\mathrm{d}}$, Gudrun Heinrich ${ }^{\mathrm{e}}$, Stefan Karg $^{\mathrm{f}}$, Nikolas Kauer ${ }^{\mathrm{g}}$, Tobias Kleinschmidt ${ }^{\mathrm{e}}$, Eric Pilon ${ }^{\mathrm{d}}$, Thomas Reiter ${ }^{\mathrm{h}}$, Jürgen Reuter ${ }^{\mathrm{c}}$, Mark Rodgers $^{\mathrm{e}}$, Ioan Wigmore ${ }^{\mathrm{a}}$

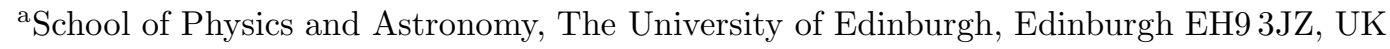

${ }^{\mathrm{b}}$ Department of Physics, University of Illinois at Urbana-Champaign, Urbana IL, 61801, USA

${ }^{\mathrm{c} P h y s i k a l i s c h e s ~ I n s t i t u t, ~ A l b e r t-L u d w i g s-U n i v e r s i t a ̈ t, ~} 79104$ Freiburg, Germany

${ }^{\mathrm{d}}$ LAPTH, 74941 Annecy le Vieux Cedex, France

eIPPP, University of Durham, Durham DH1 3LE, UK

${ }_{\mathrm{f}}^{\mathrm{f}}$ Institut für Theoretische Teilchenphysik und Kosmologie, RWTH Aachen University, 52056 Aachen, Germany

'Department of Physics, Royal Holloway, University of London, Egham TW20 0EX, UK

${ }^{\mathrm{h}}$ Nikhef, 1098 XG Amsterdam, The Netherlands

We report on the current status of the Golem project which aims at the construction of a general one-loop evaluator for matrix elements. We construct the one-loop matrix elements from Feynman diagrams in a highly automated way and provide a library for the reduction and numerically stable evaluation of the tensor integrals involved in this approach. Furthermore, we present applications to physics processes relevant for the LHC.

\section{Introduction}

Precise predictions for signals and backgrounds at high energy colliders are required to be able to identify and study New Physics in current experiments. Processes with multi-particle final states will play a significant role in this task, in particular at the LHC, which emphasises the need for predictions at next-to-leading order (NLO) for such processes.

One of the challenges of NLO calculations is the numerically stable evaluation and integration of the virtual corrections. Both the development of new unitarity based methods and the improvement of traditional methods based on Feynman diagrams have led to important new NLO results, in particular to physical predictions for $2 \rightarrow 4$ processes at hadron colliders [1/2/3/4/5/6/7/8/9|10|11]. A recent overview can also be found in [12[13].

As these calculations are technically involved, it is important to spend some effort on two features: automatisation and modularity. The latter allows for a combination of automated programs to calculate the virtual corrections with existing tools for tree level matrix element generation, phase space integration and automated tools for the subtraction of soft/collinear singularities from real radiation via a standard interface [14.

The Golem approach to the calculation of oneloop amplitudes puts particular emphasis on automatisation, as the setup can be used for massless as well as massive particles and all types of spin structures. The Feynman diagrammatic approach implies that the rational parts of the amplitudes can be evaluated at no extra cost. The 
reduction of the tensor integrals is done seminumerically, thus avoiding large algebraic expressions. A special choice of the integral basis preserves numerical stability in phase space regions with small Gram determinants. More details about the method are given in sections 2 and 3.

The Golem method in combination with automated tools for the real radiation part has been used to obtain results for e.g. $q \bar{q} \rightarrow b \bar{b} b \bar{b}$, $p p \rightarrow Z Z+$ jet and $p p \rightarrow$ graviton + jet, which will be described in sections 5 and 6 .

\section{Overview of the Algorithm}

For the consistent description of a cross-section for a $2 \rightarrow N$ particle process at NLO one needs to include the square of the tree level matrix element $A_{N}^{(0) \dagger} A_{N}^{(0)}$, the real emission of an additional massless particle $A_{N+1}^{(0) \dagger} A_{N+1}^{(0)}$ and the virtual corrections consisting of the interference term of oneloop and tree-level diagrams of the $2 \rightarrow N$ process, $A_{N}^{(0) \dagger} A_{N}^{(1)}+$ h.c.

The Golem project focuses on the efficient and numerically stable computation of the virtual corrections, as the other contributions can be added by interfacing with existing tools through a well defined generic interface [14]. The one-loop amplitude $A_{N}^{(1)}$ is represented as the helicity projections of a sum of Feynman diagrams. Each diagram $\mathcal{G}_{a}$ contributes to one or more colour structures and can be written as a sum over tensor integrals:

$$
\begin{aligned}
\mathcal{G}_{a}= & \sum_{c \in \text { colour }}|c\rangle \sum_{p=0}^{r} T_{c ; p}^{\mu_{1} \ldots \mu_{p}} \times \\
& \int \frac{\mathrm{d}^{n} q}{(2 \pi)^{n}} \frac{q_{\mu_{1}} \cdots q_{\mu_{p}}}{\prod_{j}\left(\left(q+r_{j}\right)^{2}-m_{j}^{2}+i \delta\right)}
\end{aligned}
$$

In our standard approach we use a Lorentz invariant decomposition of the tensor integrals to separate the tensor structure from the associated integral form factors. The fully contracted tensor structure is expressed in terms of spinor products and the constants of the physics model, whereas the Feynman parameter integrals are expressed in terms of form factors which can either be reduced further or be evaluated numerically. An implementation of these form factors is available in the library golem95. 15,17.

As an alternative approach one provides the numerator function

$$
\mathcal{N}_{c}\left(\hat{q}, \mu^{2}\right)=\sum_{p=0}^{r} T_{c ; p}^{\nu_{1} \ldots \nu_{p}}\left(\mu^{2}\right) \hat{q}_{\nu_{1}} \cdots \hat{q}_{\nu_{p}},
$$

where $q^{2}=\hat{q}^{2}-\mu^{2}$, as an input to some reduction at the integrand level [181920|2122], where $\mathcal{N}_{c}$ is evaluated for different numerical values of $\mu^{2}$ and the four dimensional, complex vector $\hat{q}$.

\section{Reduction of the Tensor Integrals}

The reduction of one-loop integrals follows an algorithm which has been developed in 15/16, valid for an arbitrary number of legs and both massive and massless particles. The ultraviolet and infrared divergences are regulated by dimensional regularisation.

This algorithm has been implemented as a Fortran95 library, golem95, for integrals with up to six external momenta [17. The library is publicly available at lappweb.in2p3.fr/lapth/Golem/golem95.html, together with detailed documentation and example programs.

The algebraic reduction of higher rank fourpoint and three-point functions to expressions containing only scalar integrals necessarily leads to inverse Gram determinants appearing in the coefficients of those scalar integrals. These determinants can become arbitrarily small in some phase space regions and can therefore hamper a numerically stable evaluation of the one-loop amplitude upon phase space integration. In order to deal with such situations in an automated way, our algorithm is able to avoid the occurrence of inverse Gram determinants completely. This is done by testing the value of the determinant and, in the case when it is smaller than a certain value, stopping the reduction before such inverse determinants are generated. In these cases, the tensor integrals, corresponding to integrals with Feynman parameters in the numerator, are evaluated by means of numerical integration. The use of one-dimensional integral representations guarantees a fast and stable evaluation. For integrals 
with internal masses, the option to evaluate the tensor integrals numerically prior to reduction, in regions where the Gram determinant tends to zero, is not yet supported, but is under construction. For the IR finite scalar box and triangle functions with internal masses, we use the expressions from the program OneLOop 23. The inclusion of an option to allow complex masses is underway.

To summarise, the library contains the following features:

- all tensor coefficients up to rank six sixpoint integrals, for massless as well as massive integrals

- all scalar boxes, triangles, bubbles, tadpoles

- boxes in $n+2$ dimensions up to rank three $(n=4-2 \epsilon)$ and in $n+4$ dimensions up to rank one, and triangles in $n+2$ dimensions up to rank one (larger ranks of the higher dimensional integrals are not needed in the reduction).

- automated detection of small Gram determinants and fast (one-dimensional) numerical integration of tensor integrals to avoid the occurrence of small inverse determinants

- option to calculate the rational parts only.

\section{Automated Construction of One- Loop Amplitudes}

For the automated construction of the matrix elements we have developed a package (golem-2.0 24]) combining QGRAF 25] as a diagram generator with the symbolic manipulation program Form 26] for the algebraic simplification of the generated amplitude. The expressions for the helicity projections of each diagram contributing to the process are further processed by haggies [27] to produce optimised Fortran 95 code for a fast numerical evaluation. A large part of the infrastructure and the user interface is implemented as a Python program.

For the reduction of the tensor integrals golem-2. 0 supports two choices. One can express the amplitude in terms of integral form factors as defined in 15 and implemented in golem95 [17. Alternatively, the program can generate the numerator of each Feynman diagram in a form suitable as input for Samurai [22. For each of the two options the amount of human intervention is limited to setting up an appropriate configuration file.

In order to further improve the applicability of the program we are currently implementing an interface to FeynRules 28] model files. Interoperability with existing Monte Carlo programs will be achieved by providing an interface conforming to the Binoth-Les Houches Accord [14 and some additional functionality to provide the relevant information about the matrix element to the POWHEG BOX 29].

The described features offer a great flexibility to the potential user; the achieved performance of the matrix element code and its modularity allow for a wide range of applications.

\section{Results for $q \bar{q} \rightarrow b \bar{b} b \bar{b}$}

We have used the setup described in Section 4 for generating a Fortran 95 implementation of the one-loop matrix element for the process $q \bar{q} \rightarrow$ $b \bar{b} b \bar{b}$ [7, which is one of the channels of the full $b \bar{b} b \bar{b}$ cross-section at the LHC.

The QCD induced contribution to the $4 b$ signature constitutes an important background for Higgs searches in the MSSM, where for certain parameter regions the heavy Higgs boson decays predominantly into $b \bar{b}$ pairs [1330. Also outside the framework of the MSSM interesting physics scenarios such as certain hidden valley models lead to collider signatures with many $b$ quarks 31 .

We have ensured the correctness of our oneloop matrix element by comparison to a second, independent setup using FeynArts 32, Form 26. and Maple as a tool chain. Integrated results have been obtained by three different setups:

- the virtual matrix element obtained from golem-2.0 has been used as a stand-alone program to reweight unweighted leading order events generated by WHIZARD 3334 . All tree like parts and the infrared subtrac- 
tion terms as defined in 35] using a slicing parameter [36] have been implemented in WHIZARD.

- the virtual matrix element from golem-2.0 has been linked into MadEvent 37] together with the tree-like parts from MadGraph 38. and the dipoles from MadDipole [3940].

- the third setup is the same as the previous but using Samurai [22] in place of golem95 [17] for the reduction of the numerators of the virtual matrix element.

The results use LHC kinematics $(\sqrt{s}=14 \mathrm{TeV}$ centre of mass energy). As we calculate observables for well separated jets, we cluster the final state particles by applying the $k_{T}$-algorithm 41 . into $b$-jets, which then have to pass the set of cuts,

$$
\begin{aligned}
p_{T}\left(b_{j}\right) & >30 \mathrm{GeV}, \\
\left|\eta\left(b_{j}\right)\right| & <2.5 \\
\Delta R\left(b_{i}, b_{j}\right) & >0.8 .
\end{aligned}
$$

We use the parton density functions CTEQ6.5 [42] with two-loop running of $\alpha_{s}$. The central renormalisation scale is $\mu_{0}=$ $\frac{1}{2} \sqrt{\sum_{j=1}^{4}\left[p_{T}\left(b_{j}\right)\right]^{2}}$ while the factorisation scale has been fixed at $\mu_{F}=100 \mathrm{GeV}$. The initial state includes $q \in\{u, d, s, c\}$; for the whole calculation we use the approximation $m_{b}=0$ and $m_{t} \rightarrow \infty$.

During phase space integration numerical stability was monitored by checking the magnitude of the $K$-factor for a given phase space point and also the quality of the cancellation of the infrared poles. In the worst case about $0.5 \%$ of the events needed evaluation in quadruple precision. By using the setup with Samurai, on top of previous tests we could also use several reconstruction tests as described in 22 for estimating the numerical quality for each phase space point.

Figure 1 shows the distribution for the invariant mass of the hardest pair of $b$-jets, where the jets are ordered by their $p_{T}$. The error bands are obtained by a variation of the renormalisation scale $\mu_{R}=x \mu_{0}$ for $x \in\left[\frac{1}{2} ; 4\right]$. The dashed line corresponds to the leading order result at $\mu_{R}=\mu_{0}$. As expected, the plot shows a clear reduction of the scale dependence.

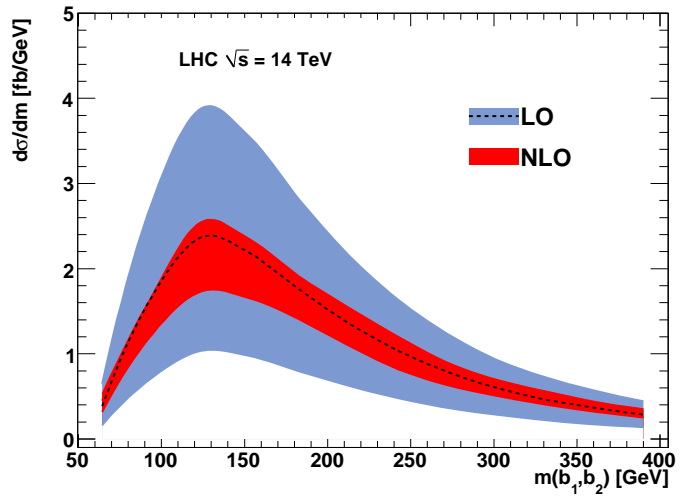

Figure 1. Distribution of the invariant mass of the hardest $b$-jet pair in $q \bar{q} \rightarrow b \bar{b} b \bar{b}$. The error bands represent a variation of the renormalisation scale $\mu_{R}=x \mu_{0}$ for $x \in\left[\frac{1}{2} ; 4\right]$.

In Figure 2 we examine the precision of $10^{5}$ phase space points by three different criteria. Since the cancellation of the single and double poles on their own are not a reliable indicator for numerical problems we also consider the local $K$ factor as a heuristic criterion (see [43]). In the chosen setup with MadEvent as an integrator and Samurai for the reduction of the amplitude the number of unstable points in double precision is below one per mil.

\section{Results for $p p \rightarrow V V+$ jet and $p p \rightarrow G+$ jet}

Golem tensor reduction methods have also been used to compute fully-differential NLO QCD corrections to $Z Z+$ jet [44] and Kaluza-Klein graviton + jet [45] production at the LHC and Tevatron. At these colliders, graviton+jet production is an important channel for graviton searches and $Z Z+$ jet production is an important background for Higgs particle and new physics searches. In both calculations, we have identified the renormalisation and factorisation scales: $\mu_{R}=\mu_{F}=$ $\mu$. In Figs. 3 and 4 we show the scale dependence of LHC cross sections for $Z Z+$ jet and graviton+jet production, respectively. The cen- 


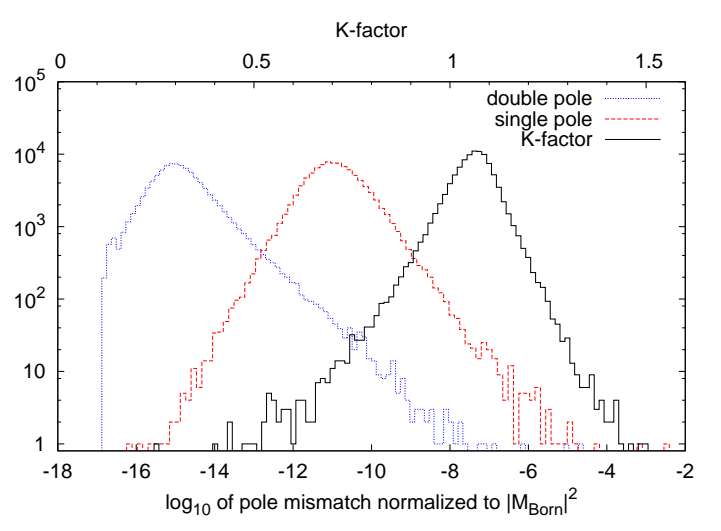

Figure 2. Criteria used for the determination of numerical stability of the virtual matrix element: cancellation of the infrared poles (lower $x$-axis) and pointwise $K$-factor (upper $x$-axis) for $10^{5}$ events. The virtual part has been evaluated using Samurai in double precision.

tral choice for $\mu$ is the $Z$ mass and the transverse momentum of the graviton $p_{T}^{G}$, respectively. The graviton search selection requires $p_{T}^{\text {miss }}>500$ $\mathrm{GeV}$ and jets are required to satisfy $p_{T}^{j}>50 \mathrm{GeV}$ and $\left|\eta_{j}\right|<4.5$. For $Z Z+$ jet production, we require $p_{T}>50 \mathrm{GeV}$ for the hardest jet. Following Ref. [4], in Fig. 3 we also show the NLO cross section when 2-jet events with a second hardest jet with $p_{T}>50 \mathrm{GeV}$ are vetoed. The jet veto clearly reduces the residual scale variation. We employed the CS dipole subtraction [35] implementations in MadDipole 39] and SHERPA 47] to calculate numerical results for the finite real corrections contribution. For both calculations internal cross checks and comparisons have been carried out (see [4445]). Our $Z Z+$ jet calculation has also been validated through a tuned comparison with the independent calculation of Ref. [48]. For a fixed phase-space point, the virtual corrections, obtained using different calculational techniques, agree at the level of $10^{-8}$ or better. The comparison of full NLO cross sections for the LHC and Tevatron, shows agreement at the per mil level. Details are reported in Ref. [12].

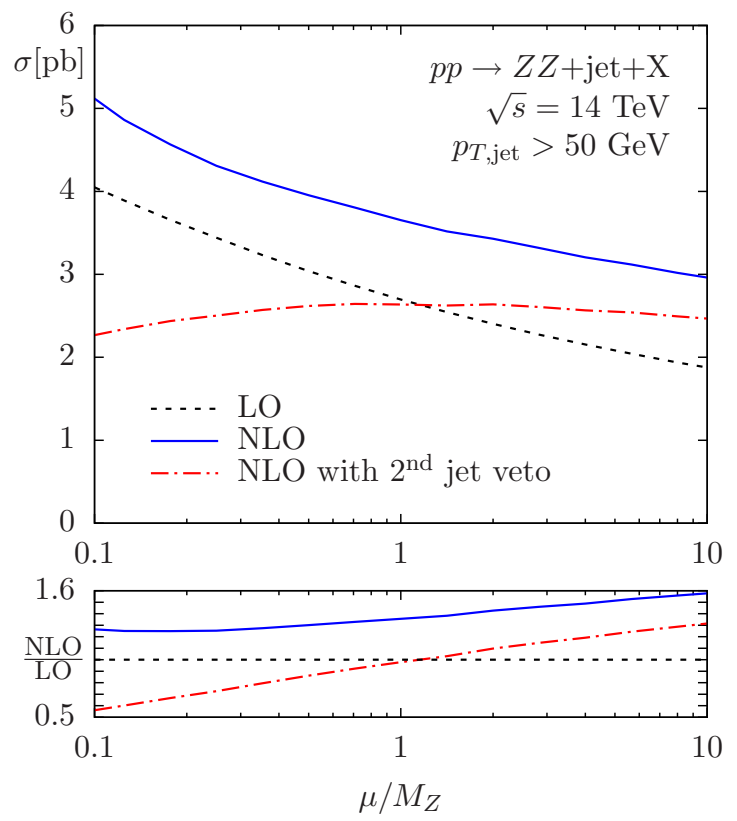

Figure 3. Scale dependence $\left(\mu_{R}=\mu_{F}=\mu\right)$ of the $Z Z+$ jet cross section at the LHC $(\sqrt{s}=14$ $\mathrm{TeV}$ ) with $p_{T}$, jet $>50 \mathrm{GeV}$ for the hardest jet in LO (dotted) and NLO (solid). The exclusive NLO cross section when a $p_{T \text {,jet }}>50 \mathrm{GeV}$ veto for additional jets is applied is also shown (dotdashed).

\section{Conclusion}

We have given an overview of the recent developments in the Golem collaboration whose main goals are the automatisation of the computation of one-loop matrix elements and the provision of precise predictions for LHC observables. As an integral part of this work we developed a library (golem95) of form factors for the reduction of oneloop tensor integrals, which in its latest version contains all integrals for one to six propagators up to full rank both for massless and massive particles in dimensional regularisation. We have implemented the Golem method for computing one-loop amplitudes in a highly automated framework (golem-2.0) which has been applied to the 


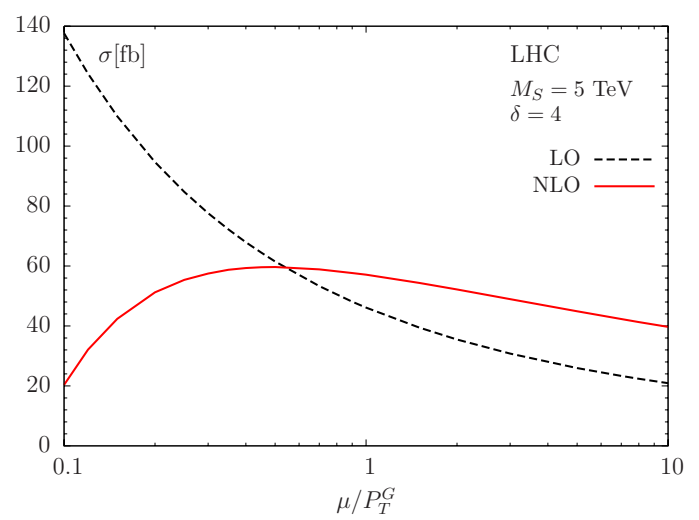

Figure 4. Scale dependence $\left(\mu_{R}=\mu_{F}=\mu\right)$ of the graviton+jet cross section at the LHC $(\sqrt{s}=14$ $\mathrm{TeV}$ ) in LO (dotted) and NLO (solid) in a KaluzaKlein model with $\delta=4$ extra dimensions and a fundamental scale $M_{S}=5 \mathrm{TeV}$. Selection cuts are described in the main text.

computation of the quark-induced channel of the process $q \bar{q} \rightarrow b \bar{b} b \bar{b}$. A similar method using the Golem tensor reduction has been used to compute the production of $Z$-pairs associated with a jet and the production of a graviton plus one jet at the LHC.

\section{Acknowledgements}

N.G. has been supported by the U. S. Department of Energy under contract No. DE-FG0291ER40677. G.H., T.K. and M.R. have been supported by the British Science and Technology Facilities Council (STFC). N.K. gratefully acknowledges the support of the IPPP Durham through an Associateship. T.R. has been supported by the Foundation FOM, project FORM 07PR2556. J.R. has been partially supported by the Ministery of Science and Culture (MWK) of the German state Baden-Württemberg.

\section{REFERENCES}

1. A. Bredenstein, A. Denner, S. Dittmaier and S. Pozzorini, JHEP 0808 (2008) 108
arXiv:0807.1248 [hep-ph]].

2. C. F. Berger et al., Phys. Rev. Lett. 102 (2009) 222001 [arXiv:0902.2760 [hep-ph]].

3. A. Bredenstein, A. Denner, S. Dittmaier and S. Pozzorini, Phys. Rev. Lett. 103 (2009) 012002 [arXiv:0905.0110 [hep-ph]].

4. R. Keith Ellis, K. Melnikov and G. Zanderighi, Phys. Rev. D 80 (2009) 094002 arXiv:0906.1445 [hep-ph]].

5. C. F. Berger et al., Phys. Rev. D 80 (2009) 074036 [arXiv:0907.1984 [hep-ph]].

6. G. Bevilacqua, M. Czakon, C. G. Papadopoulos, R. Pittau and M. Worek, JHEP 0909 (2009) 109 arXiv:0907.4723 [hep-ph]].

7. T. Binoth, N. Greiner, A. Guffanti, J. Reuter, J. P. Guillet and T. Reiter, Phys. Lett. B 685 (2010) 293 arXiv:0910.4379.

8. G. Bevilacqua, M. Czakon, C. G. Papadopoulos and M. Worek, Phys. Rev. Lett. 104 (2010) 162002 arXiv:1002.4009.

9. C. F. Berger et al., arXiv:1004.1659,

10. A. Bredenstein, A. Denner, S. Dittmaier and S. Pozzorini, JHEP 1003 (2010) 021 arXiv:1001.4006.

11. R. K. Ellis, K. Melnikov and G. Zanderighi, JHEP 0904 (2009) 077 arXiv:0901.4101 [hep-ph]].

12. J. R. Andersen et al. [SM and NLO Multileg Working Group], arXiv:1003.1241.

13. Z. Bern et al. [NLO Multileg Working Group], arXiv:0803.0494 [hep-ph].

14. T. Binoth et al., arXiv:1001.1307.

15. T. Binoth, J. P. Guillet, G. Heinrich, E. Pilon and C. Schubert, JHEP 0510 (2005) 015 arXiv:hep-ph/0504267.

16. T. Binoth, J. P. Guillet and G. Heinrich, Nucl. Phys. B $\mathbf{5 7 2}$ (2000) 361 arXiv:hep-ph/9911342.

17. T. Binoth, J. P. Guillet, G. Heinrich, E. Pilon and T. Reiter, Comput. Phys. Commun. 180 (2009) 2317 arXiv:0810.0992 [hep-ph]].

18. G. Ossola, C. G. Papadopoulos and R. Pittau, Nucl. Phys. B $\mathbf{7 6 3} \quad$ (2007) 147 arXiv:hep-ph/0609007.

19. G. Ossola, C. G. Papadopoulos and R. Pittau, JHEP 0707 (2007) 085 arXiv:0704.1271 [hep-ph]].

20. G. Ossola, C. G. Papadopoulos and R. Pit- 
tau, JHEP 0803 (2008) 042 arXiv:0711.3596 [hep-ph]].

21. P. Mastrolia, G. Ossola, C. G. Papadopoulos and R. Pittau, JHEP 0806 (2008) 030 arXiv:0803.3964 [hep-ph]].

22. P. Mastrolia, G. Ossola, T. Reiter and F. Tramontano, arXiv:1006.0710

23. A. van Hameren, C. G. Papadopoulos and R. Pittau, JHEP 0909 (2009) 106 arXiv:0903.4665 [hep-ph]].

24. T. Binoth et al., arXiv:1001.4905 [hep-ph].

25. P. Nogueira, J. Comput. Phys. 105 (1993) 279.

26. J. A. M. Vermaseren, arXiv:math-ph/0010025

27. T. Reiter, Comput. Phys. Commun. 181 (2010) 1301 arXiv:0907.3714 [hep-ph]].

28. N. D. Christensen and C. Duhr, Comput. Phys. Commun. 180 (2009) 1614 arXiv:0806.4194 [hep-ph]].

29. S. Alioli, P. Nason, C. Oleari and E. Re, JHEP 1006 (2010) 043 arXiv:1002.2581.

30. R. Lafaye, D. J. . Miller, M. Mühlleitner and S. Moretti, arXiv:hep-ph/0002238.

31. W. Krolikowski, arXiv:0803.2977 [hep-ph].

32. T. Hahn, Comput. Phys. Commun. 140 (2001) 418 arXiv:hep-ph/0012260.

33. W. Kilian, T. Ohl and J. Reuter, arXiv:0708.4233 [hep-ph].

34. M. Moretti, T. Ohl and J. Reuter, arXiv:hep-ph/0102195.

35. S. Catani and M. H. Seymour, Nucl. Phys. B 485 (1997) 291 [Erratum-ibid. B 510 (1998) 503] arXiv:hep-ph/9605323.

36. Z. Nagy and Z. Trocsanyi, Phys. Lett. B 634 (2006) 498 arXiv:hep-ph/0511328.

37. F. Maltoni and T. Stelzer, JHEP 0302 (2003) 027 arXiv:hep-ph/0208156.

38. T. Stelzer and W. F. Long, Comput. Phys. Commun. $81 \quad$ (1994) 357 arXiv:hep-ph/9401258.

39. R. Frederix, T. Gehrmann and N. Greiner, JHEP 0809 (2008) 122 arXiv:0808.2128 [hep-ph]].

40. R. Frederix, T. Gehrmann and N. Greiner, JHEP 1006, 086 (2010) arXiv:1004.2905 [hep-ph]].

41. G. C. Blazey EST al., arXiv:hep-ex/0005012.
42. W. K. Tung, H. L. Lai, A. Belyaev, J. Pumplin, D. Stump and C. P. Yuan, JHEP 0702, 053 (2007) arXiv:hep-ph/0611254.

43. T. Reiter, PhD thesis, Edinburgh (2008), arXiv:0903.0947 [hep-ph].

44. T. Binoth, T. Gleisberg, S. Karg, N. Kauer and G. Sanguinetti, Phys. Lett. B 683 (2010) 154 arXiv:0911.3181 [hep-ph]].

45. S. Karg, M. Kramer, Q. Li and D. Zeppenfeld, Phys. Rev. D 81 (2010) 094036 arXiv:0911.5095 [hep-ph]].

46. S. Dittmaier, S. Kallweit and P. Uwer, Phys. Rev. Lett. $100 \quad$ (2008) 062003 arXiv:0710.1577 [hep-ph]].

47. T. Gleisberg and F. Krauss, Eur. Phys. J. C 53 (2008) 501 arXiv:0709.2881 [hep-ph]]; F. Krauss, R. Kuhn and G. Soff, JHEP 0202 (2002) 044 arXiv:hep-ph/0109036; T. Gleisberg, S. Hoche, F. Krauss, M. Schonherr, S. Schumann, F. Siegert and J. Winter, JHEP 0902 (2009) 007 arXiv:0811.4622 [hep-ph]].

48. S. Dittmaier, S. Kallweit and P. Uwer, in preparation; S. Kallweit, PhD thesis, LudwigMaximilians-Universität Munich (2008). 